\title{
Clinicopathological and Prognostic Significance of Cancer Antigen 15-3 and Carcinoembryonic Antigen in Breast Cancer: A Meta-Analysis including 12,993 Patients
}

\author{
Xuan Li $(\mathbb{D}$, Danian Dai, Bo Chen, Hailin Tang, Xiaoming Xie $\mathbb{D}$, and Weidong Wei \\ Department of Breast Oncology, Sun Yat-sen University Cancer Center, State Key Laboratory of Oncology in South China, \\ Collaborative Innovation Center for Cancer Medicine, 651 East Dongfeng Road, Guangzhou, 510060, China
}

Correspondence should be addressed to Xiaoming Xie; xiexm@sysucc.org.cn and Weidong Wei; wwdzsdx@foxmail.com Received 11 September 2017; Accepted 30 January 2018; Published 2 May 2018

Academic Editor: Michael Gibson

Copyright ( 2018 Xuan Li et al. This is an open access article distributed under the Creative Commons Attribution License, which permits unrestricted use, distribution, and reproduction in any medium, provided the original work is properly cited.

\begin{abstract}
Purpose. The prognostic role of serum cancer antigen 15-3 (CA15-3) and carcinoembryonic antigen (CEA) in breast cancer remains controversial. In this study, we conducted a meta-analysis to investigate the prognostic value of these two markers in breast cancer patients. Methods. After electronic databases were searched, 36 studies (31 including information regarding CA153 and 23 including information regarding CEA) with 12,993 subjects were included. Based on the data directly or indirectly from the available studies, the hazard ratios (HRs) and odds ratios (ORs) and their 95\% confidence intervals (CIs) were pooled according to higher or lower marker levels. Results. Elevated CA15-3 or CEA was statistically significant with poorer DFS and $\mathrm{OS}$ in breast cancer (multivariate analysis of OS: $\mathrm{HR}=2.03,95 \% \mathrm{CI} 1.76-2.33$ for CA15-3; HR $=1.79,95 \%$ CI $1.46-2.20$ for CEA; multivariate analysis of DFS: $\mathrm{HR}=1.56,95 \%$ CI $1.06-1.55$ for CA15-3; HR $=1.77,95 \%$ CI $1.53-2.04$ for CEA). Subgroup analysis showed that CA15-3 or CEA had significant predictive values in primary or metastasis types and different cut-offs and included sample sizes and even the study publication year. Furthermore, elevated CA15-3 was associated with advanced histological grade and younger age, while elevated CEA was related to the non-triple-negative tumor type and older age. These two elevated markers were all associated with a higher tumor burden. Conclusions. This meta-analysis showed that elevated serum CA15-3 or CEA was associated with poor DFS and OS in patients with breast cancer, and they should be tested anytime if possible.
\end{abstract}

\section{Introduction}

Breast cancer has the highest incidence rate and second highest mortality rate among female cancers [1]; thus, its survival prognosis concerns doctors and patients. Prognostic factors are those clinicopathological parameters that are associated with tumor outcomes. In breast cancer, the most used prognostic factors include patient characteristics (age and menstrual status), tumor features (tumor size, node status, and TNM stage), tumor tissue markers (estrogen receptor, progesterone receptor, HER-2 status, and ki-67 status), and genetic markers (BRCA1/2) [2-8]. Using prognostic factors alone or combined to predict a worse outcome of patients and take advanced treatment early may improve patient survival. Identifying more available and convenient factors is very important.

Serum markers can be easily achieved, and they play an important role in many malignant tumors [9-11], but their role in breast cancer remains controversial. There is some correlation between tumor markers and tumor clinicopathology [12], and when the acquisition of tissue specimens is not available, in some cases, these markers may offer useful information about the phenotype of the breast cancer at an early stage [13]. Serum tumor markers in breast cancer include cancer antigen 15-3 (CA15-3), cancer antigen 27.29 (CA27.29), carcinoembryonic antigen (CEA), tissue polypeptide antigen (TPA), circulating extracellular domain of HER-2, and tissue polypeptide-specific antigen (TPS) [14, 15]. 
Among these, CA15-3 and CEA are the most used and recommended markers. The prognostic value of CA15-3 had been approved by some studies [16-18], while other studies reported negative results [19-21]. Ebeling et al. in a study of 1046 patients reported CA15-3 in univariate but not in multivariate analysis to be a predictor of a worse outcome [22]. In a review article, Duffy collected at least 10 studies and descriptively indicated that higher CA15-3 may be associated with a poor outcome but did not perform pooled analysis to confirm the results [14]. CEA is less widely investigated as a prognostic factor than CA15-3 because it has a less positive rate and is more controversial. Some studies have reported that CEA is not a predictor in primary and metastasis breast cancer $[20,21,23,24]$, but others have reported that high concentrations of CEA were related to a poor prognosis in breast cancer $[17,25,26]$. These above contradictory results of CA15-3 and CEA in breast cancer regarding their prognostic value may be due to small sample sizes, variable study designs, or other biases in each single study. We searched and combined available studies in this metaanalysis to explore the prognostic role of CA15-3 and CEA in breast cancer as well as their relationship with tumor clinicopathological factors, hoping to help medical workers affirm and properly use these two serum markers.

\section{Materials and Methods}

2.1. Search Strategy. We performed a systematic literature search using PubMed, Embase, and Web of Science. The search strategy terms are as follows: "CA15-3" (e.g., "cancer antigen 15-3," "carbohydrate antigen 15-3," and "cancer associated antigen 15-3"), "CEA" (e.g., "carcinoembryonic antigen," "carcino-embryonic antigen," and "carcino embryonic antigen"), "prognosis" (e.g., "outcome," "survival," "prognostic," "mortality," and "recurrence"), and "breast cancer" (e.g., "breast neoplasms" and "breast carcinoma"). The search was updated to July 15, 2017. The relevant articles were also manually checked.

2.2. Inclusion and Exclusion Criteria. The inclusion criteria for this meta-analysis were as follows: (i) the diagnosis of breast cancer was defined based on pathological results, (ii) CEA and CA15-3 were derived from a serum test, (iii) the correlation of serum CEA and/or CA15-3 with overall survival (OS) and/or disease-free survival (DSS) and/or disease-free survival (DFS) and/or progression-free survival (PFS) was reported, and (iv) hazard ratios (HRs) and 95\% confidence intervals (CIs) can be directly or indirectly received. The exclusion criteria were as follows: (i) abstracts, letters, reviews, case reports, proficient opinions; (ii) literature not written in English; (iii) studies without hazard ratios (HRs) and 95\% CIs; (iv) studies with duplicate data with few cases; and (v) nonhuman studies.

2.3. Data Extraction. We extracted data by two independent authors and through discussion or consensus with a third author regarding controversial data. The following items were recorded from each study: name of the first author, publication year, country, data inclusion criteria of each study, overall sample size, mean age and follow-up time of patients, tumor data of the TNM stage, time of serum sample collection, cut-off values of CEA and/or CA15-3 and the associated clinicopathological factors, and survival data, including HRs with 95\% CIs. We used the Newcastle-Ottawa Scale (NOS) to assess the quality of each study by two authors. The scores included three parts: the selectivity of patients (0-4), comparability of groups (0-2), and assessment of outcome $(0-3)$. Studies with scores $>5$ were considered high quality.

2.4. Statistical Analysis. We directly obtained all multivariate data and some univariate data of HRs and 95\% CIs from the literature. Few univariate data were estimated according to the methods illustrated by Parmar et al. and Tierney et al. using Engauge 4.0 and an HR calculation spreadsheet [27, 28]. $H R>1$ indicated a worse prognosis in breast cancer patients. All the studies with HRs and 95\% CI were classified into three groups (studies with primary TNMs I-III to group 1; studies with metastatic breast cancer to group 2; and studies with primary TNMs I-IV or unclear stage into group 3), and the pooled results included all the three study groups. The relationship between CEA and/or CA15-3 with clinicopathological parameters was determined using ORs and 95\% CIs.

In this meta-analysis, Cochran's $Q$ test was undertaken to assess the heterogeneity. All the studies first used fixed-effect (Mantel-Haenszel method) models to calculate the pooled results, but if (heterogeneity $p$ value) $\mathrm{Ph}<0.1$ or $I^{2}>50 \%$, which suggested significant heterogeneity, we changed to a more conservative random-effect (DerSimonian-Laird method) model to confirm the results. Subgroup analysis was conducted to explore and explain the heterogeneity. Publication bias was first assessed by Begg's funnel plot, and the result of $\mathrm{pr}>|z|>0.05$ was regarded as no publication bias. If bias was not certain, Egger's bias indicator test was used to reconfirm. Results with $p<0.05$ were considered statistically significant, and all the results were two sided.

\section{Results}

3.1. Study Characteristics. The initial search information retrieved 751 articles. After careful inspection, 36 studies published between 1986 and 2017 that comprised 12,993 breast cancer patients met our inclusion criteria and were finally included in this meta-analysis [3, 13, 16-21, 23-26, 29-52]. The specific processes of the literature selection are shown in Figure 1. Among them, 18 studies were from Europe and the United States, 14 studies were from Asia, and 4 studies were from Africa. Twenty-eight studies concerned the initial treatment of breast cancer patients, and eight were focused on metastatic breast cancer patients. Twenty-four studies collected serum specimens before treatment, and twelve studies collected serum specimens after surgery or at the time of recurrence and metastases. Thirty-one studies provided the survival information of serum CA15-3, and its cut-off values ranged from 20.11 to $77 \mathrm{U} / \mathrm{ml}$; meanwhile, twenty-three studies provide the survival information of serum CEA, and its 


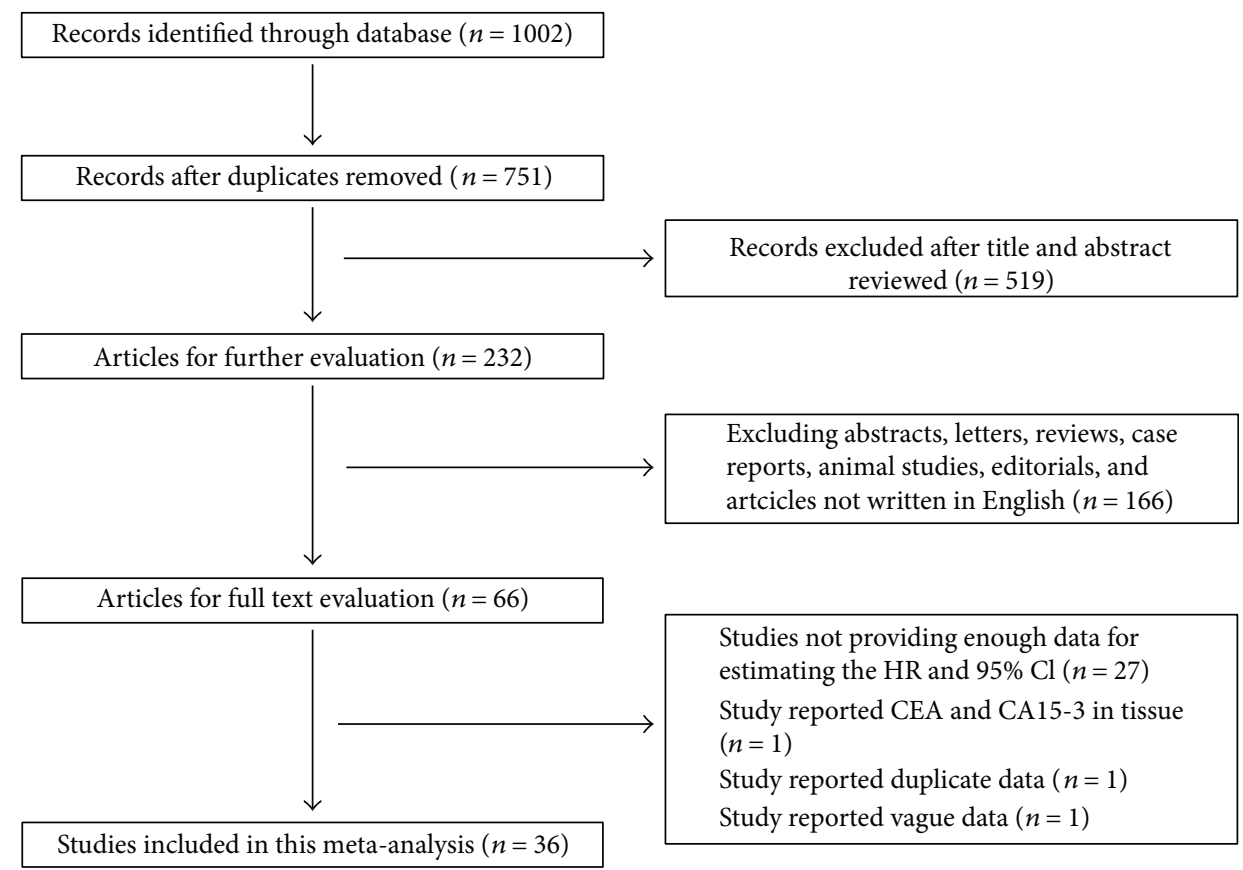

FIgURE 1: Flow chart of the included studies.

cut-off values ranged from 2.5 to $20 \mathrm{ng} / \mathrm{ml}$. Clinicopathology information correlated with serum CA15-3 or CEA could be extracted from sixteen studies. Further information of articles in this study is shown in Table 1.

3.2. CA15-3 and DFS in Breast Cancer. Fourteen studies provided univariate $\mathrm{HRs}$ and $95 \%$ CIs of DFS according to the cut-off value of serum CA15-3 (Figure 2(a)). Combined data from the fourteen studies showed that higher CA15-3 was significantly corrected with poor DFS with a pooled univariate $\mathrm{HR}$ of 2.61 (95\% CI: 2.17-3.13) with significant heterogeneity $\left(I^{2}=50 \%, \mathrm{Ph}=0.02\right.$, random effects model $)$. Ten studies provided multivariate $\mathrm{HR}$ and $95 \% \mathrm{CI}$ information of DFS concerning CA15-3 ( $\mathrm{HR}=1.56,95 \% \mathrm{CI}: 1.38-1.76$, $I^{2}=31 \%, \mathrm{Ph}=0.16$, fixed-effect model; Figure 3(a)). Only one study was included in group 2, and it showed that CA15-3 was not an independent prediction factor for DFS in metastatic breast cancer (group 2: $\mathrm{HR}=1.28 ; 95 \% \mathrm{CI}$ $0.43-3.80 ; p=0.65)$. Among the fourteen studies, two studies reported the results of PFS that was the result of group 2, which showed that HR was 2.00 (95\% CI: 1.46-2.74) for PFS without significant heterogeneity $\left(I^{2}=0 \%, \mathrm{Ph}=0.69\right)$. In multivariate subgroup analysis, the results in European countries and the United States, compared with those in Asian and African countries, showed significant subgroup heterogeneity and lower cut-off values in pretreatment patients had higher prognostic values (Table 2).

3.3. CA15-3 and OS in Breast Cancer. Twenty-one studies had extractable univariate OS HR data concerning CA15-3 (Figure 2(b)). Elevated CA15-3 indicated a shorter OS (HR $=2.86,95 \% \mathrm{CI}: 2.31-3.54, p<0.001)$ with obvious heterogeneity $\left(I^{2}=72 \%, \mathrm{Ph}<0.001\right.$, random-effect model). Heterogeneity was significantly different among the three groups as the pooled HR was the lowest in group 2 (group 1: $\mathrm{HR}=2.95,95 \%$ CI $2.28-3.82, I^{2}=22 \%, \mathrm{Ph}=0.26$; group 2: $\mathrm{HR}=1.79,95 \%$ CI $1.51-2.12, I^{2}=0 \%, \mathrm{Ph}=0.66$; group 3: $\mathrm{HR}=3.87,95 \%$ CI $\left.2.74-5.46, I^{2}=74 \%, \mathrm{Ph}=0.0003\right)$. Stratification by ethnicity found that CA15-3 was a negative predictor for OS in both European countries and the United States $(\mathrm{HR}=2.35,95 \% \mathrm{CI}: 1.74-3.18, p<0.001)$ and in Asian and African countries $(\mathrm{HR}=3.50,95 \% \mathrm{CI}: 2.59-4.71$, $p<0.001)$. Fifteen studies reported multivariate data of OS (Figure 3(b)). Elevated CA15-3 was an independent prognosis predictor for OS in breast cancer with a merged HR of 2.03 (95\% CI: 1.76-2.33, $p<0.001$ ), and heterogeneity was not significant $\left(I^{2}=0 \%, \mathrm{Ph}=0.45\right)$. Among these three groups, no significant heterogeneity was seen. The subgroup analysis results are shown in Table 2.

3.4. CEA and DFS in Breast Cancer. The univariate HR of ten studies and multivariate HR of nine studies of CEA and DFS could be obtained. Pooled univariate data showed that high CEA was associated with worse DFS with $\mathrm{HR}=2.60$ (95\% CI: 2.23-3.04; no significant heterogeneity $I^{2}=0 \%$, $\mathrm{Ph}=0.91$, fixed-effect model; Figure 2(c)). The metaanalysis of multivariate data indicated that CEA was an independent predictor for DFS with a HR of 1.77 and the $95 \% \mathrm{CI}$ ranging from 1.53 to 2.04 (no significant heterogeneity $I^{2}=0 \%, \mathrm{Ph}=0.82$, fixed-effect model; Figure $\left.3(\mathrm{c})\right)$. There was no heterogeneity among the three groups in either univariate or multivariate analysis.

3.5. CEA and OS in Breast Cancer. Meta-analysis of sixteen univariate $\mathrm{HR}$ results showed that breast cancer patients with higher CEA levels were significantly associated with shorter OS (HR $=2.46,95 \% \mathrm{CI}: 1.93-3.15, p<0.001$; heterogeneity: $I^{2}=70 \%, \mathrm{Ph}<0.001$; random-effect model; Figure $\left.2(\mathrm{~d})\right)$. 


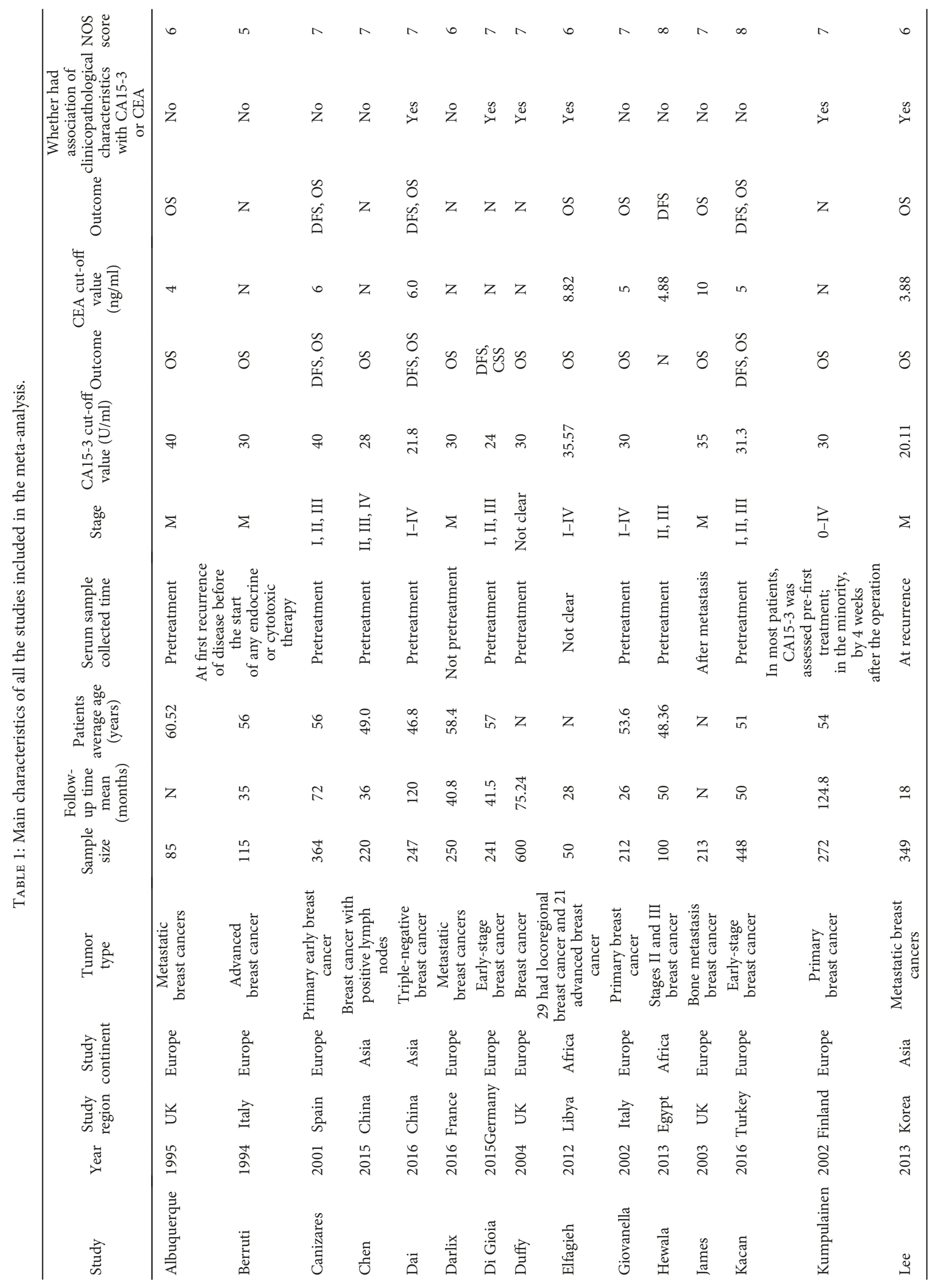




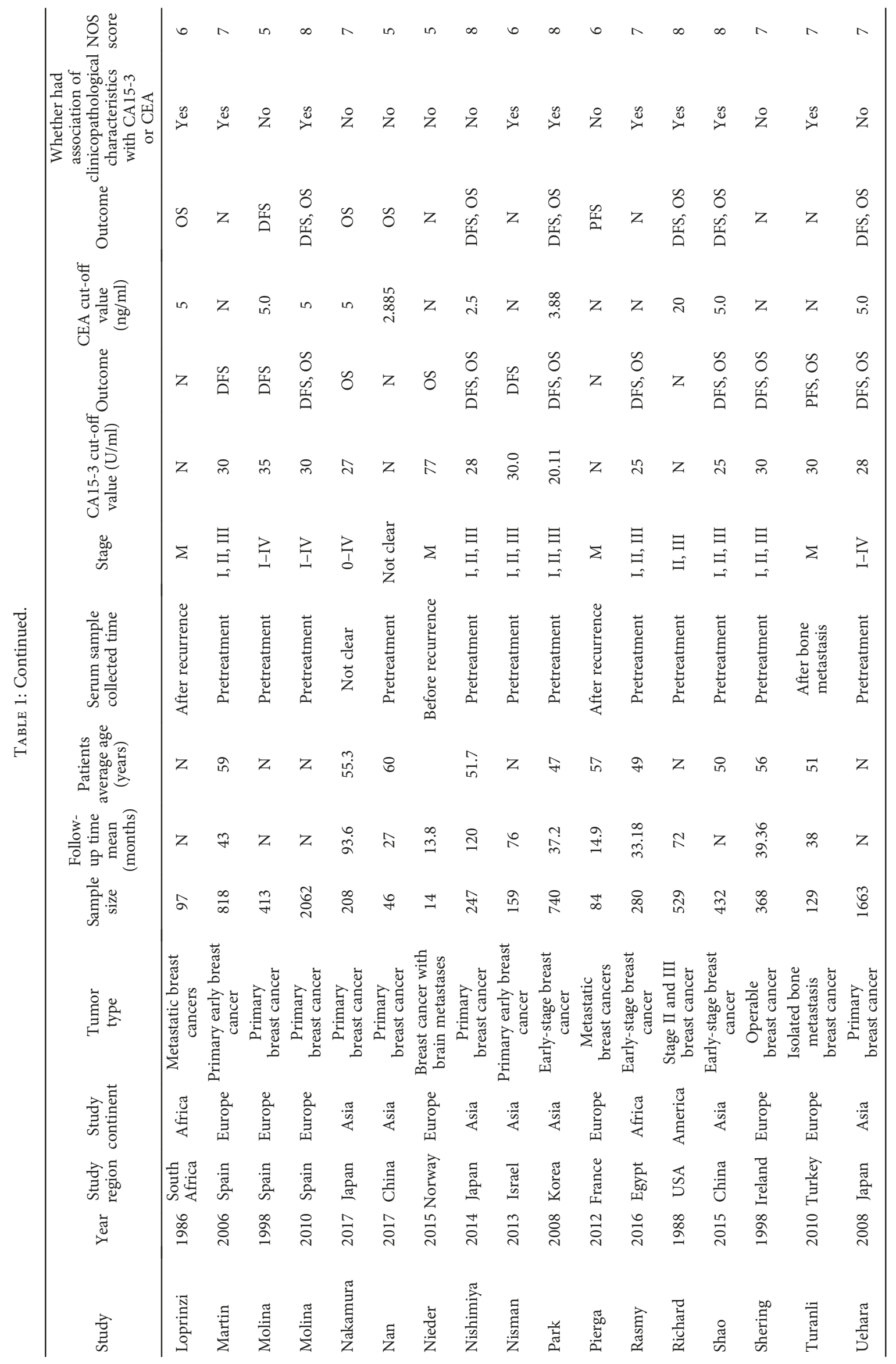




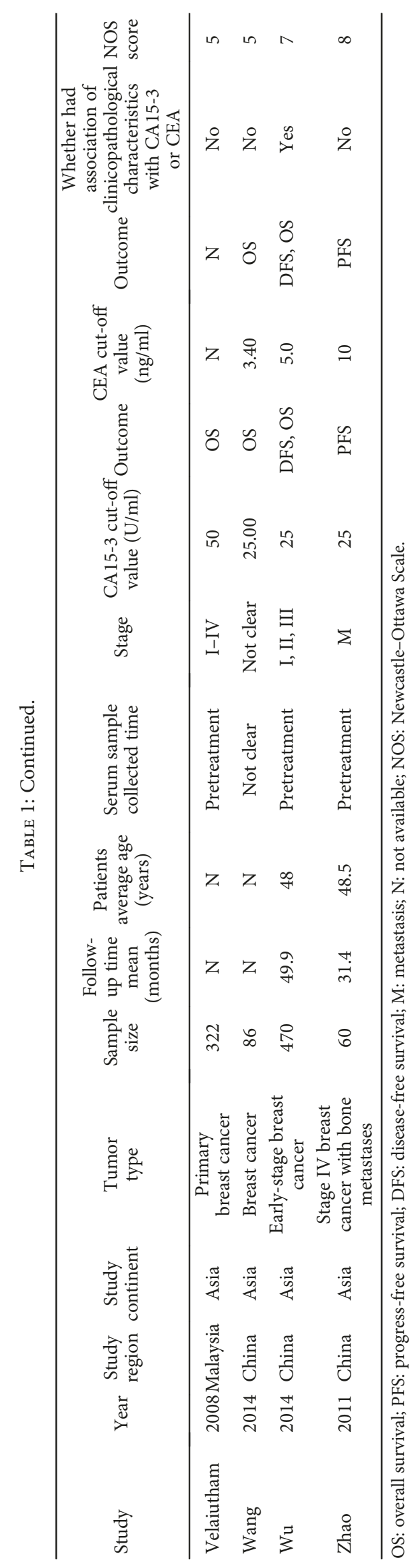




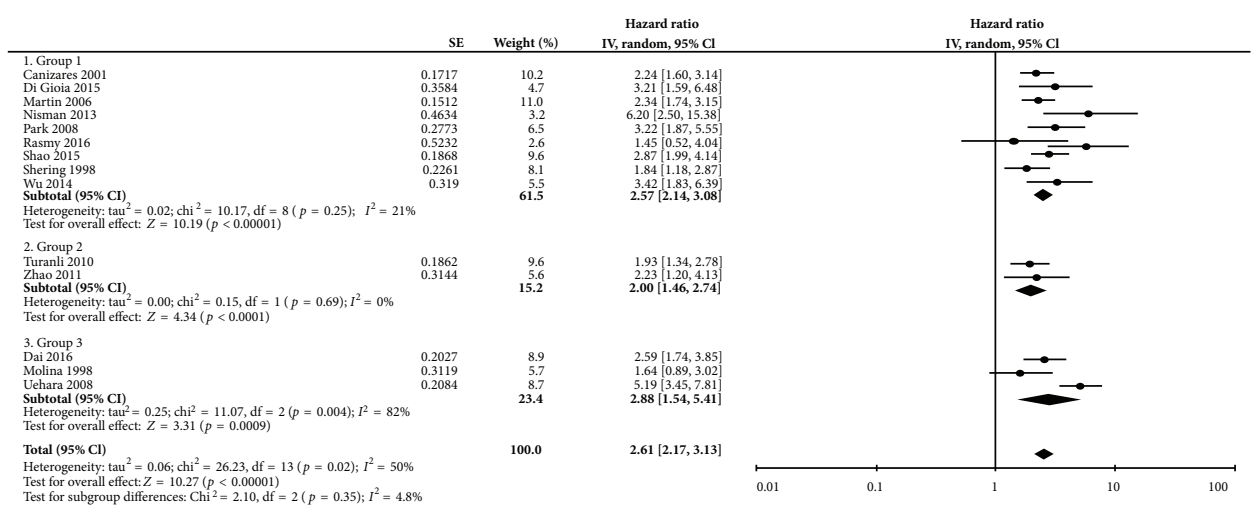

(a)

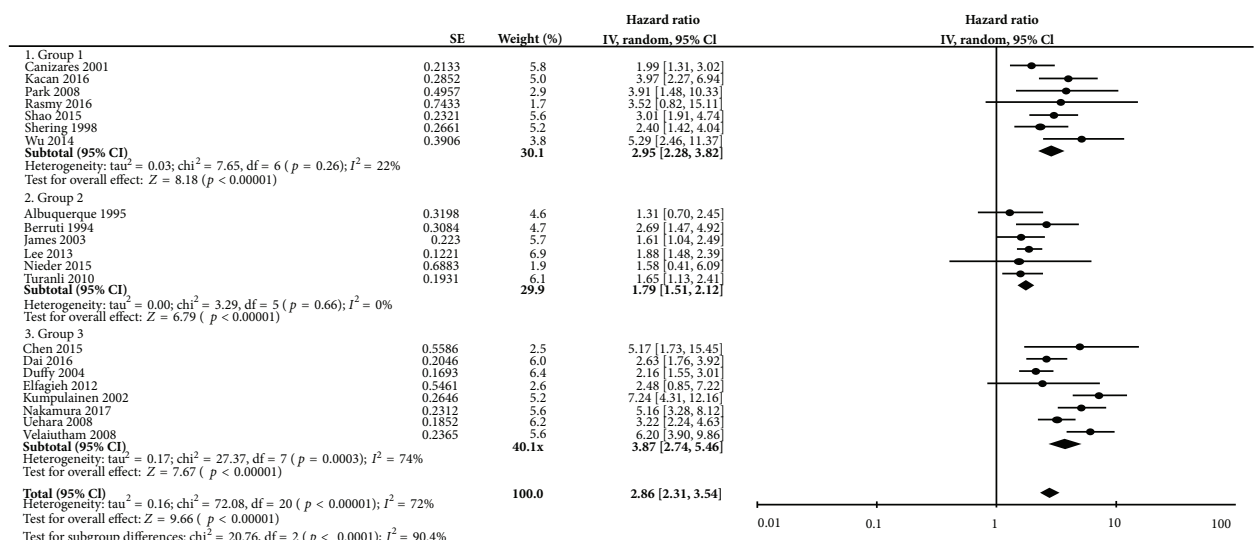

(b)

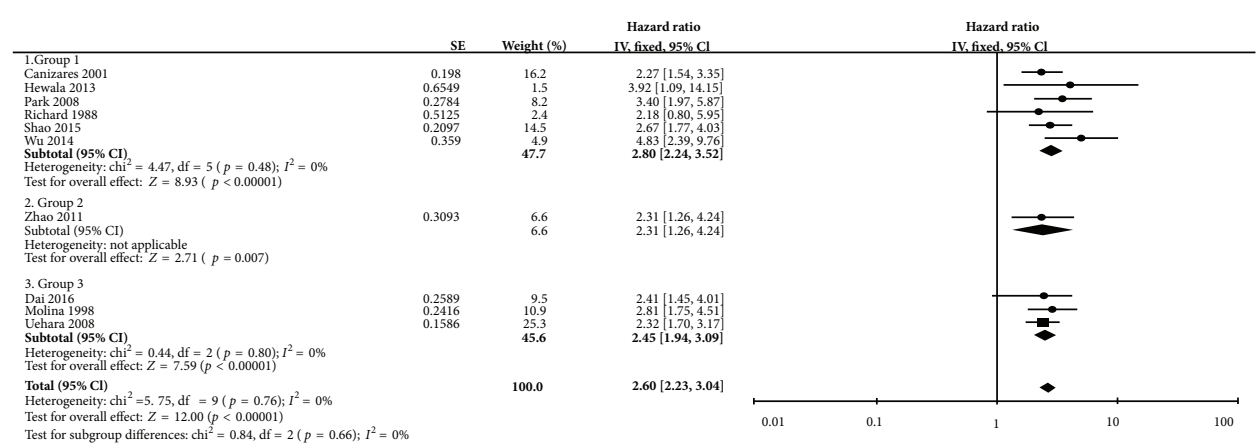

(c)

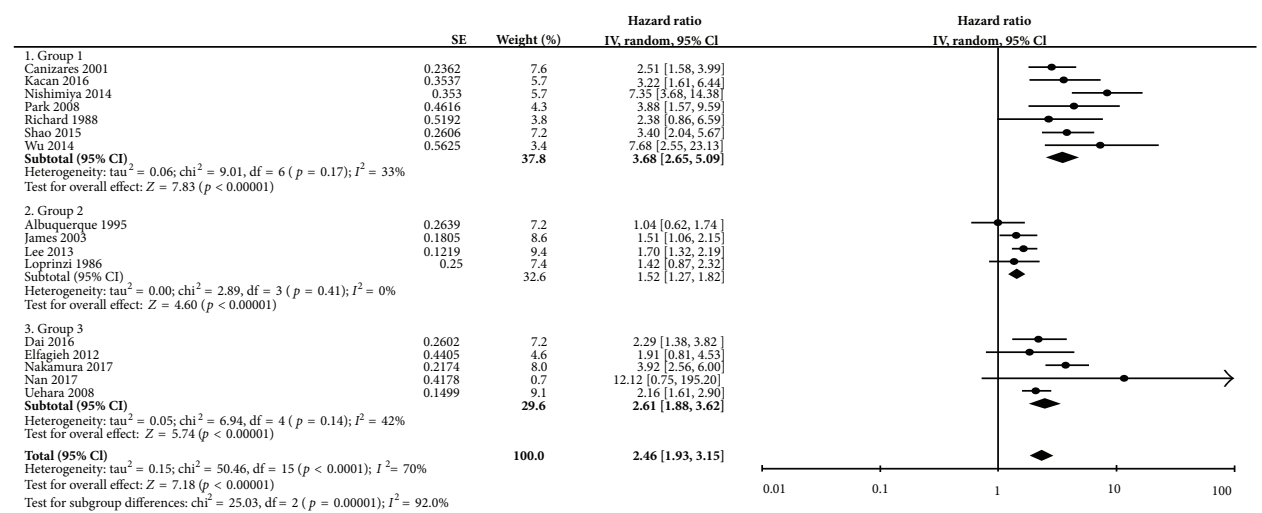

(d)

FIGURE 2: Meta-analysis of the univariate result associations between CA15-3/CEA and OS/DFS of breast cancer. The results are presented as an individual and pooled HR and 95\% CI: (a) DFS of CA15-3; (b) OS of CA15-3; (c) DFS of CEA; (d) OS of CEA. 


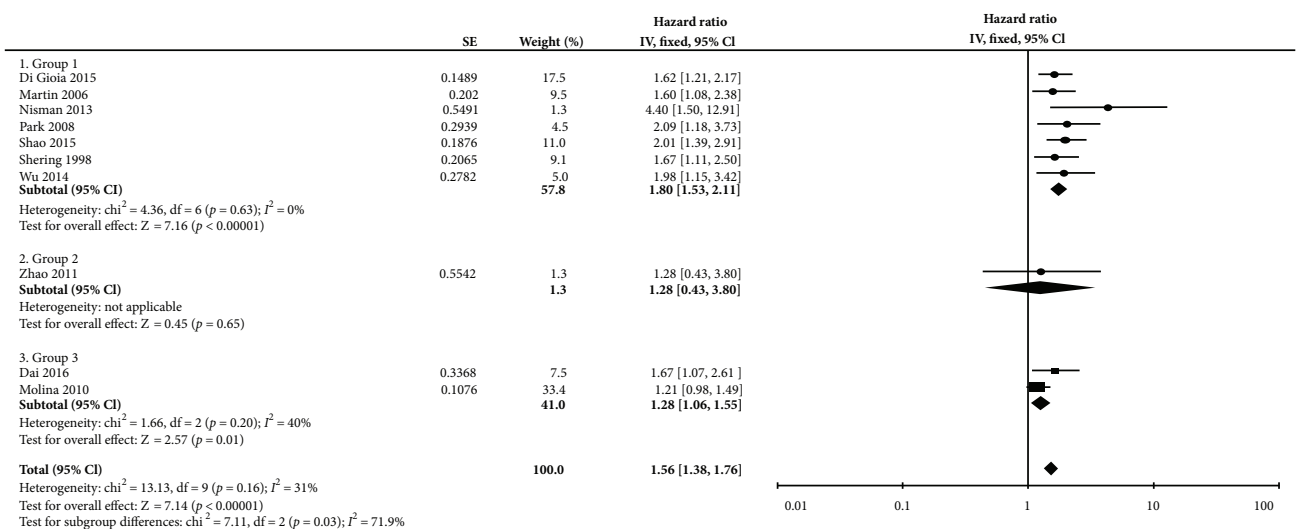

(a)

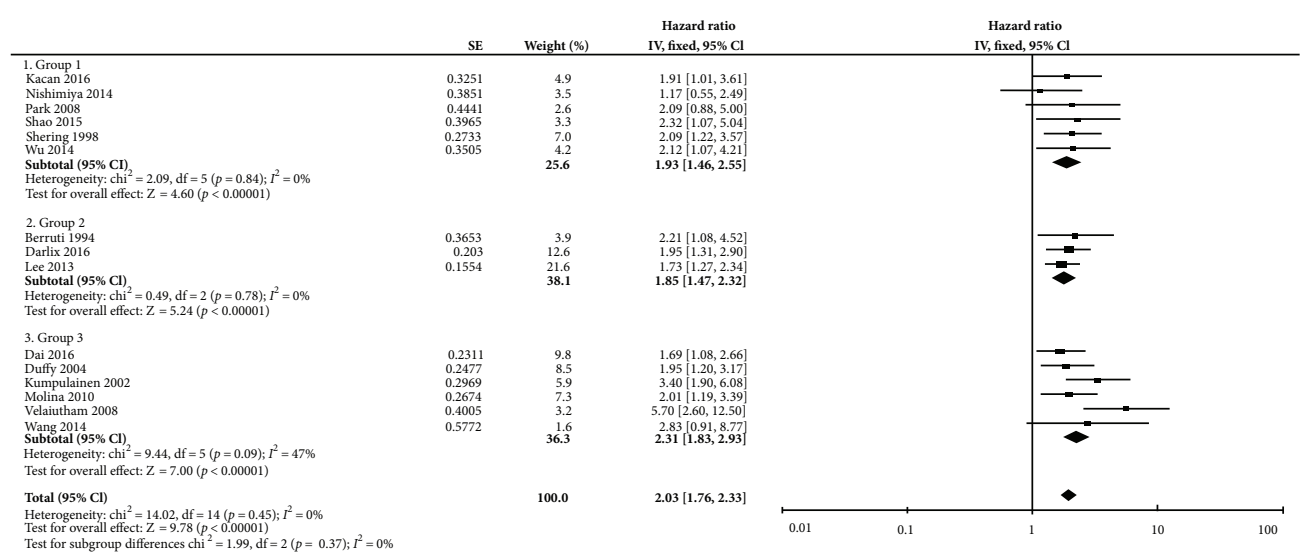

(b)

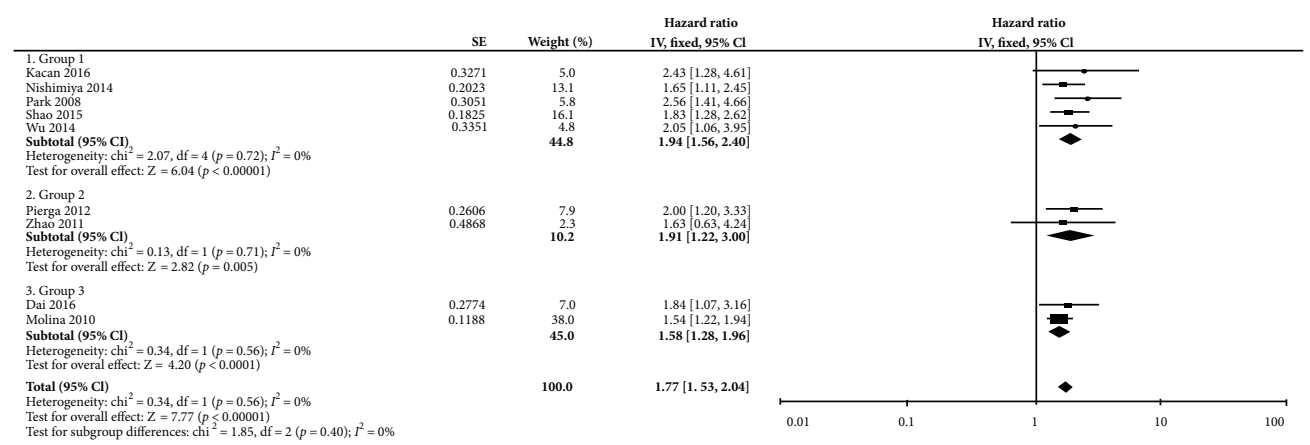

(c)

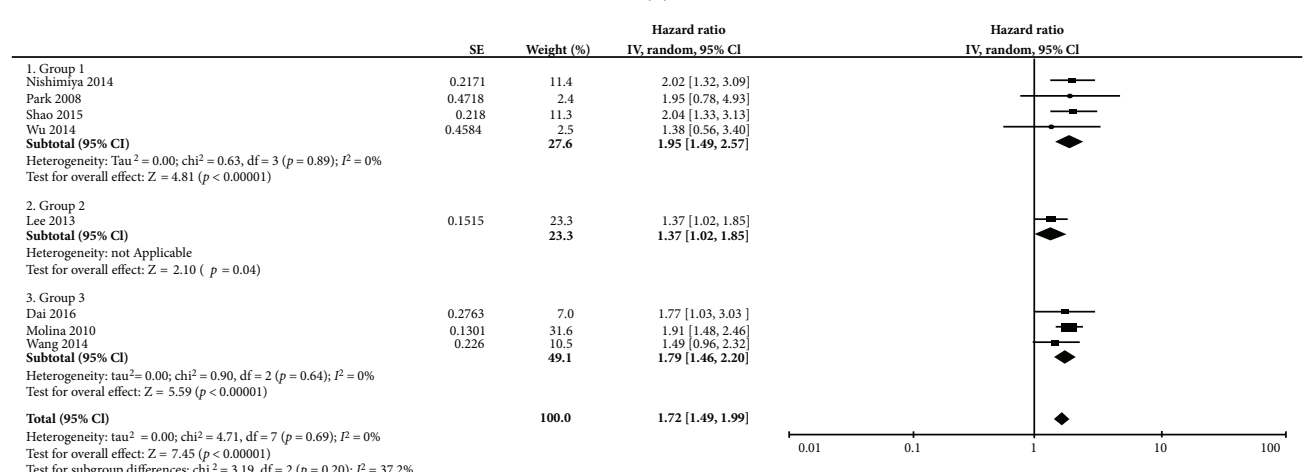

(d)

FIGURE 3: Meta-analysis of the multivariate result associations between CA15-3/CEA and OS/DFS of breast cancer. The results are presented as an individual and pooled HR and 95\% CI: (a) DFS of CA15-3; (b) OS of CA15-3; (c) DFS of CEA; (d) OS of CEA. 
Classification according to the three groups can partly explain the heterogeneity of the pooled results as each group showed no significant heterogeneity but subgroup heterogeneity differences were significant (group 1: $\mathrm{HR}=3.68,95 \%$ CI 2.65-5.09, $I^{2}=33 \%, \mathrm{Ph}=0.17$; group 2 : $\mathrm{HR}=1.52,95 \%$ CI $1.27-1.82, I^{2}=0 \%, \mathrm{Ph}=0.41$; group 3 : $\mathrm{HR}=2.61,95 \%$ CI $\left.1.88-3.62, I^{2}=42 \%, \mathrm{Ph}=0.14\right)$. Pooled multivariate $\mathrm{HR}$ results also provided similar results as higher CEA revealed an HR of 1.72 (95\% CI: 1.49-1.99) in OS without significant heterogeneity (Figure 3(d)). We performed subgroup analysis and found that, in univariate analysis, CEA derived from primary breast cancer had a higher HR than that from metastatic breast cancer. Subgroup analysis was also performed according to different cut-off values, ethnicity, and publication year (Table 2).

3.6. CA15-3 and CEA with Tumor Clinicopathological Parameters. We extracted twelve factor data according to the cut-off of the high and low groups of CA15-3 or CEA (Table 3). The results demonstrated that patients aged $\leq 35$ years had a higher rate of CA15-3 increase $(\mathrm{OR}=1.83,95 \%$ CI: $1.20-2.80, p=0.05)$, while younger ages had a lower rate of CEA rise (OR $=0.52$, 95\% CI: $0.29-0.94, p=0.03$ ). Higher rate of CA15-3 and CEA rise were correlated with tumor burden as larger tumor size, lymph node metastasis, and advanced TNM stages. Higher CA15-3 rates were also related to an advanced tumor histological grade $(\mathrm{OR}=0.63,95 \% \mathrm{CI}$ : 0.46-0.87, $p=0.005)$. However, non-triple-negative-type breast cancer was more likely to associated with a CEA level increase $(\mathrm{OR}=2.08,95 \% \mathrm{CI}: 1.30-3.33, p=0.003)$.

3.7. Publication Bias. Begg's funnel plot was used to evaluate publication bias. All the results except one showed no significant publication bias as the values of $\mathrm{pr}>|z|>0.05$. The multivariate pooled results of OS in CA15-3 had pr $>|z|=0.01$ for Begg's test. Next, we carefully examined Begg's funnel plot and performed Egger's test for this result. Egger's test showed $p>|t|=0.104$, and Begg's funnel plot was approximately symmetrical; thus, we believe that publication bias did not show a significant difference (Supplement Figure 1).

\section{Discussion}

The prognostic factors of breast cancer include tumor biopathological factors such as tumor burden, hormone receptors, HER-2, and Ki-67 levels [53, 54]. All these factors should be determined directly from tumor tissue through biopsy or surgery. It would be desirable if serum markers were used as available factors for prognosis. At present, the use of serum tumor markers in breast cancer is less well established because of its lower sensitivity and specification. Many studies have reported a low positive rate of CA15-3 and an even lower rate of CEA $[17,34]$. Without more powerful serum markers, although imperfect, CA15-3 and CEA remain the most commonly used biomarkers in breast cancer and are recommended for practical use by the American Society of Clinical Oncology (ASCO). However, because of insufficient data, the use of CA15-3 and CEA as screening, diagnostic, and staging tests to detect recurrence and monitoring the response to treatment alone is not recommended by ASCO as well as its prognostic functions [15]. However, the European Group on Tumor Markers has recommended the use of CEA and CA15-3 to assess the prognosis in breast cancer [55]. Our meta-analysis was focused on serum CA15-3 and CEA prognostic ability and their best use of these two markers in breast cancer.

The meta-analysis indicated that an elevated CA15-3 level significantly corresponded with poor DFS and OS of breast cancer. In our analysis, serum CA15-3 had prognostic ability in the pretreatment of primary early-stage and primary all-stage breast cancer patients. It also showed prognostic values in metastasis in OS but not in multivariate analysis of DFS because of only one study reported this result, and it may show great bias. The reason why CA15-3 can predict the prognosis in breast cancer is not fully clear, but as CA15-3 is the soluble form of MUC1, this may be related with the function of MUC1. MUC1 was reported to not only allow the cancer cells to escape the immune system but also promote cancer cell migration by activating some membrane receptors [56-59]. Serum CA15-3 in metastasis studies had lower HR than that in primary studies. The cause may be that CA15-3 is closely corrected with tumor burden, and in pretreated tumors, it is consistently associated with tumor characteristics that can best predict the prognosis of survival [60]. Nieder et al. found that overall survival was significantly associated with CA15-3 in brain metastasis patients with breast cancer and this trend was not found in other cancers [32]. Subgroup analysis indicated that different cut-off values of pretreatment tumor studies had different predictive abilities, and the lower cut-off value studies had higher HRs in DFS and OS. However, Keshaviah et al. combined 7 study group trials and found a lower cut-off would decrease the predictive value of CA15-3 [61]. This different result may be because Keshaviah used serum CA15-3 tested at any time during disease; however, in the present study, we used the pretreatment serum sample only. We also found differences according to country. European countries and the United States had lower HRs than Asian and African countries because developed countries had improved screening methods and self-consciousness. As the tumor can be found at an early stage in developed countries [62-64], CA15-3, used as a predictor, may be more useful in developing countries, and this region-specific use of tumor marker test phenomenon was also found by Ramsey et al. [65]. Though CA15-3 seems to be able to assess the prognosis of breast cancer, there was lack of prospective testing to clarify it which limited its practical use in the clinical setting.

The CEA levels are less likely to be elevated than CA15-3; however, in symptomatic breast cancer patients, CEA sensitivity increased, and some studies found that CEA are able to correlate with the stage of disease as well as prognosis though these results are also in dispute. De Jong-Bakker et al. reported the pretreatment level of CEA had no relationship with all groups of patients regarding prognosis as the same results of other small-sample studies report $[33,34,44,66]$. However, in our pooled results, higher serum CEA had lower DFS and OS both in univariate and multivariate analyses. Serum CEA 
TABLE 2: Summary of the meta-analysis results.

\begin{tabular}{|c|c|c|c|c|c|c|c|c|c|c|}
\hline \multirow{2}{*}{ Analysis } & \multicolumn{5}{|c|}{ Univariate analysis } & \multicolumn{5}{|c|}{ Multivariate analysis } \\
\hline & $N$ & HR $(95 \%$ CI $)$ & $p$ & $I^{2}$ & $\mathrm{Ph}$ & $N$ & $\operatorname{HR}(95 \% \mathrm{CI})$ & $p$ & $I^{2}$ & $\mathrm{Ph}$ \\
\hline \multicolumn{11}{|l|}{ CA15-3 DFS } \\
\hline All & 14 & $2.61(2.17-3.13)$ & $<0.001$ & 50 & 0.02 & 10 & $1.56(1.38-1.76)$ & $<0.001$ & 31 & 0.16 \\
\hline \multicolumn{11}{|l|}{ Subgroup 1} \\
\hline Primary early-stage breast cancer & 9 & $2.57(2.14-3.08)$ & $<0.001$ & 21 & 0.25 & 7 & $1.80(1.53-2.11)$ & $<0.001$ & 0 & 0.63 \\
\hline Metastatic breast cancer & 2 & $2.00(1.46-2.74)$ & $<0.001$ & 0 & 0.69 & 1 & $1.28(0.43-3.80)$ & 0.65 & - & - \\
\hline Primary all-stage or unclear-stage breast cancer & 3 & $2.88(1.54-5.41)$ & $<0.001$ & 82 & 0.004 & 2 & $1.28(1.06-1.55)$ & $<0.001$ & 40 & 0.20 \\
\hline \multicolumn{11}{|l|}{ Subgroup 2} \\
\hline Cut-off $\leq 25$ & 6 & $2.84(2.30-3.50)$ & $<0.001$ & 0 & 0.77 & 5 & $1.80(1.50-2.16)$ & $<0.001$ & 0 & 0.85 \\
\hline Cut-off $>25$ & 6 & $2.69(1.87-3.87)$ & $<0.001$ & 75 & 0.001 & 4 & $1.39(1.17-1.64)$ & $<0.001$ & 59 & 0.06 \\
\hline \multicolumn{11}{|l|}{ Subgroup 3} \\
\hline Asia and Africa & 8 & $3.18(2.46-4.11)$ & $<0.001$ & 44 & 0.08 & 6 & $1.96(1.57-2.44)$ & $<0.001$ & 0 & 0.65 \\
\hline Europe and America & 6 & $2.14(1.82-2.52)$ & $<0.001$ & 0 & 0.67 & 4 & $1.41(1.22-1.63)$ & $<0.001$ & 24 & 0.27 \\
\hline \multicolumn{11}{|l|}{ Subgroup 4} \\
\hline Sample size $<500$ & 11 & $2.37(2.00-2.81)$ & $<0.001$ & 20 & 0.26 & 7 & $1.78(1.50-2.10)$ & $<0.001$ & 0 & 0.65 \\
\hline Sample size $\geq 500$ & 3 & $3.36(2.01-5.61)$ & $<0.001$ & 79 & 0.008 & 3 & $1.35(1.13-1.61)$ & 0.001 & 50 & 0.14 \\
\hline \multicolumn{11}{|l|}{ Subgroup 5} \\
\hline Publish year $>2010$ & 7 & $2.82(2.3-3.48)$ & $<0.001$ & 0 & 0.45 & 6 & $1.80(1.50-2.17)$ & $<0.001$ & 0 & 0.53 \\
\hline Publish year $\leq 2010$ & 7 & $2.45(1.86-3.23)$ & $<0.001$ & 69 & 0.004 & 4 & $1.39(1.19-1.64)$ & $<0.001$ & 39 & 0.18 \\
\hline \multicolumn{11}{|l|}{ CA15-3 OS } \\
\hline All & 21 & $2.86(2.31-3.54)$ & $<0.001$ & 72 & $<0.0001$ & 15 & $2.03(1.76-2.33)$ & $<0.001$ & 0 & 0.45 \\
\hline \multicolumn{11}{|l|}{ Subgroup 1} \\
\hline Primary early-stage breast cancer & 7 & $2.95(2.28-3.82)$ & $<0.001$ & 22 & 0.26 & 6 & $1.93(1.46-2.55)$ & $<0.001$ & 0 & 0.84 \\
\hline Metastatic breast cancer & 6 & $1.79(1.51-2.12)$ & $<0.001$ & 0 & 0.66 & 3 & $1.85(1.47-2.32)$ & $<0.001$ & 0 & 0.78 \\
\hline Primary all-stage or unclear-stage breast cancer & 8 & $3.87(2.74-5.46)$ & $<0.001$ & 74 & $<0.0001$ & 6 & $2.31(1.83-2.93)$ & $<0.001$ & 47 & 0.09 \\
\hline \multicolumn{11}{|l|}{ Subgroup 2} \\
\hline Cut-off $\leq 25$ & 5 & $3.11(2.39-4.05)$ & $<0.001$ & 0 & 0.59 & 5 & $1.98(1.46-2.69)$ & $<0.001$ & 0 & 0.90 \\
\hline Cut-off $>25$ & 10 & $3.57(2.62-4.88)$ & $<0.001$ & 74 & $<0.0001$ & 7 & $2.24(1.79-2.80)$ & $<0.001$ & 46 & 0.09 \\
\hline \multicolumn{11}{|l|}{ Subgroup 3} \\
\hline Asia and Africa & 11 & $3.50(2.59-4.71)$ & $<0.001$ & 70 & 0.0002 & 8 & $1.93(1.58-2.35)$ & $<0.001$ & 34 & 0.16 \\
\hline Europe and America & 10 & $2.35(1.74-3.18)$ & $<0.001$ & 72 & 0.0002 & 7 & $2.13(1.74-2.60)$ & $<0.001$ & 0 & 0.81 \\
\hline \multicolumn{11}{|l|}{ Subgroup 4} \\
\hline Sample size $<500$ & 18 & $2.87(2.24-3.7)$ & $<0.001$ & 75 & $<0.0001$ & 12 & $2.03(1.74-2.38)$ & $<0.001$ & 21 & 0.23 \\
\hline Sample size $\geq 500$ & 3 & $2.72(1.96-3.78)$ & $<0.001$ & 37 & 0.20 & 3 & $1.99(1.43-2.77)$ & $<0.001$ & 0 & 0.99 \\
\hline \multicolumn{11}{|l|}{ Subgroup 5} \\
\hline Publish year $>2010$ & 10 & $3.17(2.33-4.31)$ & $<0.001$ & 62 & 0.005 & 8 & $1.82(1.52-2.18)$ & $<0.001$ & 0 & 0.90 \\
\hline Publish year $\leq 2010$ & 11 & $2.65(1.94-3.62)$ & $<0.001$ & 79 & $<0.0001$ & 7 & $2.41(1.92-3.03)$ & $<0.001$ & 21 & 0.27 \\
\hline \multicolumn{11}{|l|}{ CEA DFS } \\
\hline All & 10 & $2.60(2.23-3.04)$ & $<0.001$ & 0 & 0.91 & 9 & $1.77(1.53-2.04)$ & $<0.001$ & 0 & 0.82 \\
\hline \multicolumn{11}{|l|}{ Subgroup 1} \\
\hline Primary early-stage breast cancer & 6 & $2.80(2.24-3.52)$ & $<0.001$ & 0 & 0.48 & 5 & $1.94(1.56-2.40)$ & $<0.001$ & 0 & 0.72 \\
\hline Metastatic breast cancer & 1 & $2.31(1.26-4.24)$ & 0.007 & - & - & 2 & $1.91(1.22-3.00)$ & 0.005 & 0 & 0.71 \\
\hline Primary all-stage or unclear-stage breast cancer & 3 & $2.45(1.94-3.09)$ & $<0.001$ & 0 & 0.80 & 2 & $1.58(1.28-1.96)$ & $<0.001$ & 0 & 0.56 \\
\hline \multicolumn{11}{|l|}{ Subgroup 2} \\
\hline Cut-off $\leq 5$ & 6 & $2.77(2.29-3.37)$ & $<0.001$ & 0 & 0.48 & 6 & $1.74(1.49-2.04)$ & $<0.001$ & 0 & 0.54 \\
\hline Cut-off $>5$ & 3 & $2.31(1.72-3.10)$ & $<0.001$ & 0 & 0.98 & 1 & $1.84(1.07-3.16)$ & $<0.001$ & - & - \\
\hline \multicolumn{11}{|l|}{ Subgroup 3} \\
\hline Asia and Africa & 6 & $2.65(2.19-3.20)$ & $<0.001$ & 0 & 0.46 & 5 & $1.95(1.53-2.48)$ & $<0.001$ & 0 & 0.89 \\
\hline Europe and America & 4 & $2.50(1.89-3.32)$ & $<0.001$ & 0 & 0.80 & 4 & $1.67(1.40-2.00)$ & $<0.001$ & 0 & 0.52 \\
\hline
\end{tabular}


TABLE 2: Continued.

\begin{tabular}{|c|c|c|c|c|c|c|c|c|c|c|}
\hline \multirow{2}{*}{ Analysis } & \multicolumn{5}{|c|}{ Univariate analysis } & \multicolumn{5}{|c|}{ Multivariate analysis } \\
\hline & $N$ & HR (95\% CI) & $p$ & $I^{2}$ & $\mathrm{Ph}$ & $N$ & $\operatorname{HR}(95 \% \mathrm{CI})$ & $p$ & $I^{2}$ & $\mathrm{Ph}$ \\
\hline \multicolumn{11}{|l|}{ Subgroup 4} \\
\hline Sample size $<500$ & 6 & $2.62(2.11-3.24)$ & $<0.001$ & 0 & 0.54 & 7 & $1.87(1.54-2.26)$ & $<0.001$ & 0 & 0.97 \\
\hline Sample size $\geq 500$ & 4 & $2.59(2.06-3.25)$ & $<0.001$ & 0 & 0.64 & 2 & $1.65(1.33-2.05)$ & $<0.001$ & 59 & 0.12 \\
\hline \multicolumn{11}{|l|}{ Subgroup 5} \\
\hline Publish year $>2010$ & 5 & $2.79(2.15-3.60)$ & $<0.001$ & 0 & 0.50 & 7 & $1.87(1.54-2.26)$ & $<0.001$ & 0 & 0.97 \\
\hline Publish year $\leq 2010$ & 5 & $2.50(2.05-3.04)$ & $<0.001$ & 0 & 0.74 & 2 & $1.65(1.33-2.05)$ & $<0.001$ & 59 & 0.12 \\
\hline \multicolumn{11}{|l|}{ CEA OS } \\
\hline All & 16 & $2.46(1.93-3.15)$ & $<0.001$ & 70 & $<0.0001$ & 8 & $1.72(1.49-1.99)$ & $<0.001$ & 0 & 0.69 \\
\hline \multicolumn{11}{|l|}{ Subgroup 1} \\
\hline Primary early-stage breast cancer & 7 & $3.68(2.65-5.09)$ & $<0.001$ & 33 & 0.17 & 4 & $1.95(1.49-2.57)$ & $<0.001$ & 0 & 0.89 \\
\hline Metastatic breast cancer & 4 & $1.52(1.27-1.82)$ & $<0.001$ & 0 & 0.41 & 1 & $1.37(1.02-1.85)$ & 0.04 & - & - \\
\hline Primary all-stage or unclear-stage breast cancer & 5 & $2.61(1.88-3.62)$ & $<0.001$ & 42 & 0.14 & 3 & $1.79(1.46-2.20)$ & $<0.001$ & 0 & 0.64 \\
\hline \multicolumn{11}{|l|}{ Subgroup 2} \\
\hline Cut-off $\leq 5$ & 8 & $3.80(2.67-5.39)$ & $<0.001$ & 59 & 0.02 & 6 & $1.86(1.56-2.20)$ & $<0.001$ & 0 & 0.88 \\
\hline Cut-off $>5$ & 4 & $2.34(1.73-3.17)$ & $<0.001$ & 0 & 0.96 & 1 & $1.77(1.03-3.03)$ & $<0.001$ & - & - \\
\hline \multicolumn{11}{|l|}{ Subgroup 3} \\
\hline Asia and Africa & 11 & $2.82(2.07-3.85)$ & $<0.001$ & 72 & $<0.001$ & 7 & $1.65(1.38-1.96)$ & $<0.001$ & 0 & 0.70 \\
\hline Europe and America & 5 & $1.87(1.26-2.77)$ & 0.002 & 61 & 0.04 & 1 & $1.91(1.48-2.46)$ & $<0.001$ & - & - \\
\hline \multicolumn{11}{|l|}{ Subgroup 4} \\
\hline Sample size $<500$ & 13 & $2.48(1.84-3.34)$ & $<0.001$ & 75 & $<0.0001$ & 6 & $1.64(1.37-1.95)$ & $<0.001$ & 0 & 0.60 \\
\hline Sample size $\geq 500$ & 3 & $2.29(1.75-3.00)$ & $<0.001$ & 0 & 0.48 & 2 & $1.91(1.50-2.45)$ & $<0.001$ & 0 & 0.96 \\
\hline \multicolumn{11}{|l|}{ Subgroup 5} \\
\hline Publish year $>2010$ & 9 & $3.27(2.22-4.82)$ & $<0.001$ & 73 & $<0.0001$ & 6 & $1.64(1.37-1.95)$ & $<0.001$ & 0 & 0.60 \\
\hline Publish year $\leq 2010$ & 7 & $1.82(1.39-2.39)$ & $<0.001$ & 53 & 0.05 & 2 & $1.91(1.50-2.45)$ & $<0.001$ & 0 & 0.96 \\
\hline
\end{tabular}

N: number of studies; HR: hazard ratio; 95\% CI: 95\% confidence interval; Ph: $p$ values of $Q$ test for heterogeneity test; OS: overall survival; DFS: disease-free survival; "-" means unavailable.

showed no significant difference in HR in primary and metastasis breast cancer regarding DFS but had a higher HR of primary cancer than metastasis regarding OS. Subgroup analysis showed a lower cut-off value, and studies in Asian and African countries had higher HRs with the same tendency as CA15-3. In clinical use, CEA can be informative when levels of CA15-3 remain below the cut-off point, but for now, no high-level evidence study has verified it [67].

CA15-3 and CEA have been reported to be associated with clinicopathological parameters [68]. Both the CA15-3 and CEA levels were elevated with a larger tumor size, positive lymph node metastasis, and advanced TNM stage. A higher elevation of the markers does not indicate that their prognostic value has increased. In CA15-3 and CEA with tumor burden prognostic analysis, Molina et al. found that CEA had prognostic values in node-positive or nodenegative breast cancer, but CA15-3 showed a prognostic value only in the node-negative patients [47]. Gion et al. also found that CA15-3 was significantly associated with the prognosis in node-positive cases not in node-negative cases [69]. In patients younger than 35 years and older, the results in CA15-3 and CEA had significant differences and were reversed. Young patients are more likely to have elevated CA15-3; however, older patients are more likely to have elevated CEA. This can inspire doctors to use different cut-off values in young and older breast cancer patients to best manage young patients. The molecular types of breast cancer may also be different in tumor markers expressed. Triple-negative breast cancer may more likely show high CEA according to a study that included 247 triple-negative breast cancer patients, in which the best cut-off value using the X-Tile program was $6 \mathrm{ng} / \mathrm{ml}$ higher than the recommended $5 \mathrm{ng} / \mathrm{ml}$ [25]. $\mathrm{Li}$ et al.'s study and other studies found that the CA15-3 levels showed a significant difference according to molecular subtype $[60,70]$, but the pooled results of our study saw no difference.

Several limitations need to be carefully considered in this analysis. First, almost all the included studies in this analysis were retrospective, making them more susceptible to some biases. Though we could not exclude all potential residual confounding, the asymmetry in the funnel plots at least showed the absence of publication bias, thus maintaining the substantial consistency of the results. Second, the publication year of the included studies in this analysis ranged from 1986 to 2017. This long period led to great differences; thus, we performed subgroup analysis and used the cut-off year of 2010 to limit the heterogeneity. Third, some studies have found that CA15-3 and/or CEA only had prognostic value according to molecular tumor types, but we could not identify sufficient studies to pool the results according to different 


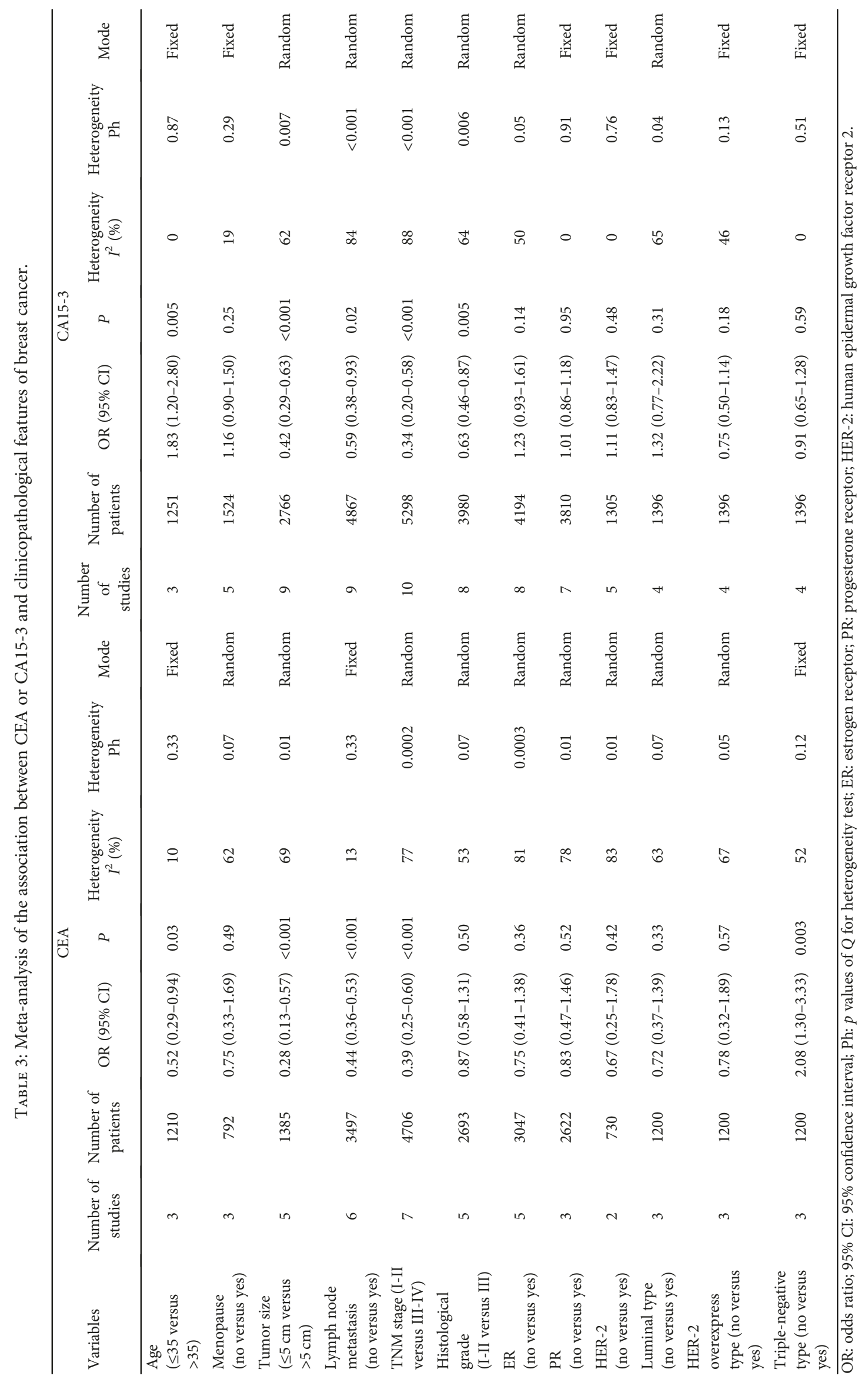


molecular types of breast cancer. Fourth, although we performed multivariate analysis of CA15-3 and CEA as prognostic factors, the multivariate analyses were adjusted to different factors in different studies; thus, the pooled results using CA15-3 and/or CEA as an independent predictor should be carefully considered.

In conclusion, we found that both CA15-3 and CEA were good prognostic factors for poor DFS and OS when abnormal levels were found in breast cancer patients. Furthermore, higher CA15-3 or CEA had more significant prognostic significance for pretreatment primary breast cancer, a cancer occurring in Asian and African countries. Elevated CA15-3 and CEA were closely associated with age and tumor burden. In the future, more studies with uniform cut-off values, large-scale data, and good designs are needed to validate our conclusion.

\section{Additional Points}

Writing Assistance. This article received English editing from American Journal Experts (certificate verification key: E6F411E9-CF02-D4A3-CEF3).

\section{Ethical Approval}

This article does not contain any studies with human participants or animals performed by any of the authors. Meta-analyses does not need ethical approval in Sun Yat-sen University Cancer Center.

\section{Conflicts of Interest}

The authors declare that they have no conflict of interest.

\section{Authors' Contributions}

Xuan Li collected, analyzed, and interpreted the data and was a major contributor in writing the manuscript. Danian Dai and Bo Chen were the second reviewers for data collection, analysis, and risk of bias assessment. Hailin Tang, Xiaoming Xie, and Weidong Wei also participated in data analysis and interpretation, as well as in manuscript preparation. All authors read and approved the final manuscript. Xuan Li, Danian Dai, and Bo Chen contributed equally to this work.

\section{Supplementary Materials}

Figure 1: Begg's funnel plot of the study: (a) univariate result of DFS of CA15-3; (b) multivariate result of DFS of CA15-3; (c) univariate result of OS of CA15-3; (d) multivariate result of OS of CA15-3; (e) univariate result of DFS of CEA; (f) multivariate result of DFS of CEA; $(\mathrm{g})$ univariate result of OS of CEA; (h) multivariate result of OS of CEA. (Supplementary Materials)

\section{References}

[1] R. L. Siegel, K. D. Miller, and A. Jemal, "Cancer statistics, 2017," CA: A Cancer Journal for Clinicians, vol. 67, no. 1, pp. 7-30, 2017.
[2] A. Goldhirsch, W. C. Wood, R. D. Gelber et al., "Progress and promise: highlights of the international expert consensus on the primary therapy of early breast cancer 2007," Annals of Oncology, vol. 18, no. 7, pp. 1133-1144, 2007.

[3] H. Nishimiya, Y. Kosaka, K. Yamashita et al., "Prognostic significance of $\mathrm{Ki}-67$ in chemotherapy-naive breast cancer patients with 10-year follow-up," Anticancer Research, vol. 34, no. 1, pp. 259-268, 2014.

[4] D. L. Page, "Prognosis and breast cancer. Recognition of lethal and favorable prognostic types," The American Journal of Surgical Pathology., vol. 15, no. 4, pp. 334-349, 1991.

[5] C. W. Elston and I. O. Ellis, "Pathological prognostic factors in breast cancer. I. The value of histological grade in breast cancer: experience from a large study with long-term follow-up," Histopathology, vol. 19, no. 5, pp. 403-410, 1991.

[6] C. L. Carter, C. Allen, and D. E. Henson, "Relation of tumor size, lymph node status, and survival in 24,740 breast cancer cases," Cancer, vol. 63, no. 1, pp. 181-187, 1989.

[7] M. J. Duffy, "Estrogen receptors: role in breast cancer," Critical Reviews in Clinical Laboratory Sciences, vol. 43, no. 4, pp. 325-347, 2006.

[8] J. S. Ross, J. A. Fletcher, G. P. Linette et al., “The HER-2/neu gene and protein in breast cancer 2003: biomarker and target of therapy," The Oncologist, vol. 8, no. 4, pp. 307-325, 2003.

[9] G. J. S. Rustin, "Use of CA-125 to assess response to new agents in ovarian cancer trials," Journal of Clinical Oncology, vol. 21, no. 90100, pp. 187s-193s, 2003.

[10] M. J. Duffy, "Carcinoembryonic antigen as a marker for colorectal cancer: is it clinically useful?," Clinical Chemistry, vol. 47, no. 4, pp. 624-630, 2001.

[11] C. Parker, "Active surveillance: towards a new paradigm in the management of early prostate cancer," The Lancet Oncology, vol. 5, no. 2, pp. 101-106, 2004.

[12] F. Lumachi, S. M. Basso, A. A. Brandes, D. Pagano, and M. Ermani, "Relationship between tumor markers CEA and CA 15-3, TNM staging, estrogen receptor rate and MIB-1 index in patients with pT1-2 breast cancer," Anticancer Research, vol. 24, no. 5B, pp. 3221-3224, 2004.

[13] D. Di Gioia, M. Dresse, D. Mayr, D. Nagel, V. Heinemann, and P. Stieber, "Serum HER2 in combination with CA 15-3 as a parameter for prognosis in patients with early breast cancer," Clinica Chimica Acta, vol. 440, pp. 16-22, 2015.

[14] M. J. Duffy, "Serum tumor markers in breast cancer: are they of clinical value?," Clinical Chemistry, vol. 52, no. 3, pp. 345-351, 2006.

[15] L. Harris, H. Fritsche, R. Mennel et al., "American Society of Clinical Oncology 2007 update of recommendations for the use of tumor markers in breast cancer," Journal of Clinical Oncology, vol. 25, no. 33, pp. 5287-5312, 2007.

[16] T. Kacan, B. Yucel, S. Bahar et al., "The prognostic value of high pretreatment plasma d-dimer levels in non-metastatic breast cancer patients with absence of venous thromboembolism," International Journal of Hematology and Oncology, vol. 26, no. 3, pp. 136-144, 2016.

[17] Y. Shao, X. Sun, Y. He, C. Liu, and H. Liu, "Elevated levels of serum tumor markers CEA and CA153 are prognostic parameters for different molecular subtypes of breast cancer," PloS One, vol. 10, no. 7, article e0133830, 2015.

[18] S. G. Shering, F. Sherry, E. W. McDermott, N. J. O'Higgins, and M. J. Duffy, "Preoperative CA 15-3 concentrations predict 
outcome of patients with breast carcinoma," Cancer, vol. 83, no. 12 , pp. 2521-2527, 1998.

[19] A. Rasmy, W. Abozeed, S. Elsamany et al., "Correlation of preoperative Ki67 and serum CA15.3 levels with outcome in early breast cancers a multi institutional study," Asian Pacific Journal of Cancer Prevention, vol. 17, no. 7, pp. 3595-3600, 2016.

[20] M. Elfagieh, F. Abdalla, A. Gliwan, J. Boder, W. Nichols, and A. Buhmeida, "Serum tumour markers as a diagnostic and prognostic tool in Libyan breast cancer," Tumour Biology, vol. 33, no. 6, pp. 2371-2377, 2012.

[21] K. V. Albuquerque, M. R. Price, R. A. Badley et al., "Pre-treatment serum levels of tumour markers in metastatic breast cancer: a prospective assessment of their role in predicting response to therapy and survival," European Journal of Surgical Oncology, vol. 21, no. 5, pp. 504-509, 1995.

[22] F. G. Ebeling, P. Stieber, M. Untch et al., "Serum CEA and CA 15-3 as prognostic factors in primary breast cancer," British Journal of Cancer, vol. 86, no. 8, pp. 1217-1222, 2002.

[23] C. L. Loprinzi, D. C. Tormey, P. Rasmussen et al., "Prospective evaluation of carcinoembryonic antigen levels and alternating chemotherapeutic regimens in metastatic breast cancer," Journal of Clinical Oncology, vol. 4, no. 1, pp. 46-56, 1986.

[24] J. Nan, J. Li, X. Li, G. Guo, X. Wen, and Y. Tian, "Preoperative serum carcinoembryonic antigen as a marker for predicting the outcome of three cancers," Biomarkers in Cancer, vol. 9, article 1179299X1769014, 2017.

[25] D. Dai, B. Chen, H. Tang et al., "Nomograms for predicting the prognostic value of pre-therapeutic CA15-3 and CEA serum levels in TNBC patients," PLoS One, vol. 11, no. 8, article e0161902, 2016.

[26] J. S. Lee, S. Park, J. M. Park, J. H. Cho, S. I. Kim, and B. W. Park, "Elevated levels of preoperative CA 15-3 and CEA serum levels have independently poor prognostic significance in breast cancer," Annals of Oncology, vol. 24, no. 5, pp. 1225-1231, 2013.

[27] M. K. B. Parmar, V. Torri, and L. Stewart, "Extracting summary statistics to perform meta-analyses of the published literature for survival endpoints," Statistics in Medicine, vol. 17, no. 24, pp. 2815-2834, 1998.

[28] J. F. Tierney, L. A. Stewart, D. Ghersi, S. Burdett, and M. R. Sydes, "Practical methods for incorporating summary timeto-event data into meta-analysis," Trials, vol. 8, no. 1, p. 16, 2007.

[29] Z. Chen, S. Xu, D. Su et al., "A new tumor biomarker, serum protein peak at 3,144 m/z, in patients with node-positive breast cancer," Clinical \& Translational Oncology, vol. 17, no. 6, pp. 486-494, 2015.

[30] A. Darlix, P. J. Lamy, E. Lopez-Crapez et al., "Serum HER2 extra-cellular domain, S100ss and CA 15-3 levels are independent prognostic factors in metastatic breast cancer patients," BMC Cancer, vol. 16, no. 1, p. 428, 2016.

[31] K. Nakamura, E. Okada, S. Ukawa et al., "Characteristics and prognosis of Japanese female breast cancer patients: the BioBank Japan project," Journal of Epidemiology, vol. 27, no. 3, pp. S58-S64, 2017.

[32] C. Nieder, A. Dalhaug, E. Haukland, B. Mannsaker, and A. Pawinski, "Tumor marker analyses in patients with brain metastases: patterns of practice and implications for survival prediction research," Tumour Biology, vol. 36, no. 8, pp. 6471-6476, 2015.
[33] G. Wang, Y. Qin, J. Zhang et al., "Nipple discharge of CA15-3, CA125, CEA and TSGF as a new biomarker panel for breast cancer," International Journal of Molecular Sciences, vol. 15, no. 12, pp. 9546-9565, 2014.

[34] S. G. Wu, Z. Y. He, J. Zhou et al., "Serum levels of CEA and CA15-3 in different molecular subtypes and prognostic value in Chinese breast cancer," Breast, vol. 23, no. 1, pp. 88-93, 2014.

[35] S. Velaiutham, N. A. Taib, K. L. Ng, B. K. Yoong, and C. H. Yip, "Does the pre-operative value of serum CA15-3 correlate with survival in breast cancer?," Asian Pacific Journal of Cancer Prevention, vol. 9, no. 3, pp. 445-448, 2008.

[36] R. Molina, J. Jo, X. Filella et al., "c-erbB-2 oncoprotein, CEA, and CA 15.3 in patients with breast cancer: prognostic value," Breast Cancer Research and Treatment., vol. 51, no. 2, pp. 109-119, 1998.

[37] A. Berruti, M. Tampellini, M. Torta, T. Buniva, G. Gorzegno, and L. Dogliotti, "Prognostic value in predicting overall survival of two mucinous markers: CA 15-3 and CA 125 in breast cancer patients at first relapse of disease," European Journal of Cancer, vol. 30, no. 14, pp. 2082-2084, 1994.

[38] J. J. James, A. J. Evans, S. E. Pinder et al., "Bone metastases from breast carcinoma: histopathological-radiological correlations and prognostic features," British Journal of Cancer, vol. 89, no. 4, pp. 660-665, 2003.

[39] T. I. Hewala and N. A. Abd El-Moneim, "Breast cancer proliferative activity: is it the source of serum free DNA?," Alexandria Journal of Medicine, vol. 49, no. 4, pp. 337-341, 2013.

[40] E. J. Kumpulainen, R. J. Keskikuru, and R. T. Johansson, “Serum tumor marker CA 15.3 and stage are the two most powerful predictors of survival in primary breast cancer," Breast Cancer Research and Treatment, vol. 76, no. 2, pp. 95-102, 2002.

[41] J. Y. Pierga, D. Hajage, T. Bachelot et al., "High independent prognostic and predictive value of circulating tumor cells compared with serum tumor markers in a large prospective trial in first-line chemotherapy for metastatic breast cancer patients," Annals of Oncology, vol. 23, no. 3, pp. 618-624, 2012.

[42] M. Uehara, T. Kinoshita, T. Hojo, S. Akashi-Tanaka, E. Iwamoto, and T. Fukutomi, "Long-term prognostic study of carcinoembryonic antigen (CEA) and carbohydrate antigen 15-3 (CA 15-3) in breast cancer," International Journal of Clinical Oncology, vol. 13, no. 5, pp. 447-451, 2008.

[43] M. J. Duffy, C. Duggan, R. Keane et al., "High preoperative CA 15-3 concentrations predict adverse outcome in node-negative and node-positive breast cancer: study of 600 patients with histologically confirmed breast cancer," Clinical Chemistry, vol. 50, no. 3, pp. 559-563, 2004.

[44] B. W. Park, J. W. Oh, J. H. Kim et al., "Preoperative CA 15-3 and CEA serum levels as predictor for breast cancer outcomes," Annals of Oncology, vol. 19, no. 4, pp. 675-681, 2008.

[45] X. Zhao, X. Xu, Q. Zhang et al., "Prognostic and predictive value of clinical and biochemical factors in breast cancer patients with bone metastases receiving "metronomic" zoledronic acid," BMC Cancer, vol. 11, no. 1, 2011.

[46] S. Turanli and A. Cetin, "Prognostic role of serum cancer antigen 15-3 in breast cancer patients with isolated bone metastases," Biomarkers, vol. 15, no. 5, pp. 418-423, 2010.

[47] R. Molina, J. M. Auge, B. Farrus et al., "Prospective evaluation of carcinoembryonic antigen (CEA) and carbohydrate antigen 15.3 (CA 15.3) in patients with primary locoregional breast cancer," Clinical Chemistry, vol. 56, no. 7, pp. 1148-1157, 2010. 
[48] L. Giovanella, L. Ceriani, G. Giardina, D. Bardelli, F. Tanzi, and S. Garancini, "Serum cytokeratin fragment 21.1 (CYFRA 21.1) as tumour marker for breast cancer: comparison with carbohydrate antigen 15.3 (CA 15.3) and carcinoembryonic antigen (CEA)," Clinical Chemistry and Laboratory Medicine, vol. 40, no. 3, pp. 298-303, 2002.

[49] R. L. Theriault, G. N. Hortobagyi, H. A. Fritsche, D. Frye, R. Martinez, and A. U. Buzdar, "The role of serum CEA as a prognostic indicator in stage II and III breast cancer patients treated with adjuvant chemotherapy," Cancer, vol. 63 , no. 5 , pp. 828-835, 1989.

[50] B. Nisman, O. Maimon, T. Allweis et al., "The prognostic significance of LIAISON (R) CA15-3 assay in primary breast cancer," Anticancer Research, vol. 33, no. 1, pp. 293-299, 2013.

[51] A. Martin, M. D. Corte, A. M. Alvarez et al., "Prognostic value of pre-operative serum CA 15.3 levels in breast cancer," Anticancer Research, vol. 26, no. 5B, pp. 3965-3971, 2006.

[52] F. Cañizares, J. Sola, M. Pérez et al., "Preoperative values of CA 15-3 and CEA as prognostic factors in breast cancer: a multivariate analysis," Tumor Biology, vol. 22, no. 5, pp. 273-281, 2001.

[53] C. W. Elston, I. O. Ellis, and S. E. Pinder, "Pathological prognostic factors in breast cancer," Critical Reviews in Oncology/ Hematology, vol. 31, no. 3, pp. 209-223, 1999.

[54] C. Isaacs, V. Stearns, and D. F. Hayes, "New prognostic factors for breast cancer recurrence," Seminars in Oncology, vol. 28, no. 1, pp. 53-67, 2001.

[55] R. Molina, V. Barak, A. van Dalen et al., "Tumor markers in breast cancer-European Group on Tumor Markers recommendations," Tumour Biology, vol. 26, no. 6, pp. 281-293, 2005.

[56] S. Ogata, P. J. Maimonis, and S. H. Itzkowitz, "Mucins bearing the cancer-associated sialosyl-Tn antigen mediate inhibition of natural killer cell cytotoxicity," Cancer Research, vol. 52, no. 17, pp. 4741-4746, 1992.

[57] M. J. Hudson, G. W. Stamp, K. S. Chaudhary et al., "Human MUC1 mucin: a potent glandular morphogen," The Journal of Pathology, vol. 194, no. 3, pp. 373-383, 2001.

[58] R. J. Quin and M. A. McGuckin, "Phosphorylation of the cytoplasmic domain of the MUC1 mucin correlates with changes in cell-cell adhesion," International Journal of Cancer, vol. 87, no. 4, pp. 499-506, 2000.

[59] J. A. Schroeder, M. C. Thompson, M. M. Gardner, and S. J. Gendler, "Transgenic MUC1 interacts with epidermal growth factor receptor and correlates with mitogen-activated protein kinase activation in the mouse mammary gland," The Journal of Biological Chemistry, vol. 276, no. 16, pp. 13057-13064, 2001.

[60] H. Li, K. Chen, F. Su, E. Song, and C. Gong, "Preoperative CA 15-3 levels predict the prognosis of nonmetastatic luminal A breast cancer," Journal of Surgical Research, vol. 189, no. 1, pp. 48-56, 2014.

[61] A. Keshaviah, S. Dellapasqua, N. Rotmensz et al., "CA15-3 and alkaline phosphatase as predictors for breast cancer recurrence: a combined analysis of seven International Breast Cancer Study Group trials," Annals of Oncology, vol. 18, no. 4, pp. 701-708, 2007.

[62] G. Abdoli, M. Bottai, K. Sandelin, and T. Moradi, "Breast cancer diagnosis and mortality by tumor stage and migration background in a nationwide cohort study in Sweden," Breast, vol. 31, pp. 57-65, 2017.
[63] S. Eaker, P. W. Dickman, V. Hellstrom, M. M. Zack, J. Ahlgren, and L. Holmberg, "Regional differences in breast cancer survival despite common guidelines," Cancer Epidemiology, Biomarkers \& Prevention, vol. 14, no. 12, pp. 2914-2918, 2005.

[64] S. P. Leong, Z. Z. Shen, T. J. Liu et al., "Is breast cancer the same disease in Asian and western countries?," World Journal of Surgery, vol. 34, no. 10, pp. 2308-2324, 2010.

[65] S. D. Ramsey, N. L. Henry, J. R. Gralow et al., "Tumor marker usage and medical care costs among older early-stage breast cancer survivors," Journal of Clinical Oncology, vol. 33, no. 2, pp. 149-155, 2015.

[66] M. De Jong-Bakker, A. A. Hart, J. P. Persijn, and F. J. Cleton, "Prognostic significance of CEA in breast cancer: a statistical study," European Journal of Cancer \& Clinical Oncology, vol. 17, no. 12, pp. 1307-1313, 1981.

[67] C. M. Sturgeon, M. J. Duffy, U. H. Stenman et al., "National Academy of Clinical Biochemistry laboratory medicine practice guidelines for use of tumor markers in testicular, prostate, colorectal, breast, and ovarian cancers," Clinical Chemistry, vol. 54, no. 12, pp. e11-e79, 2008.

[68] A. K. Agrawal, M. Jelen, J. Rudnicki et al., "The importance of preoperative elevated serum levels of CEA and CA15-3 in patients with breast cancer in predicting its histological type," Folia Histochemica et Cytobiologica, vol. 48, no. 1, pp. 26-29, 2010.

[69] M. Gion, P. Boracchi, R. Dittadi et al., "Prognostic role of serum CA15.3 in 362 node-negative breast cancers. An old player for a new game," European Journal of Cancer, vol. 38, no. 9, pp. 1181-1188, 2002.

[70] O. Brouckaert, A. Laenen, H. Wildiers et al., "The prognostic role of preoperative and (early) postoperatively change in CA15.3 serum levels in a single hospital cohort of primary operable breast cancers," Breast, vol. 22, no. 3, pp. 254-262, 2013. 


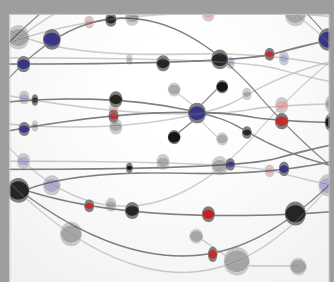

The Scientific World Journal
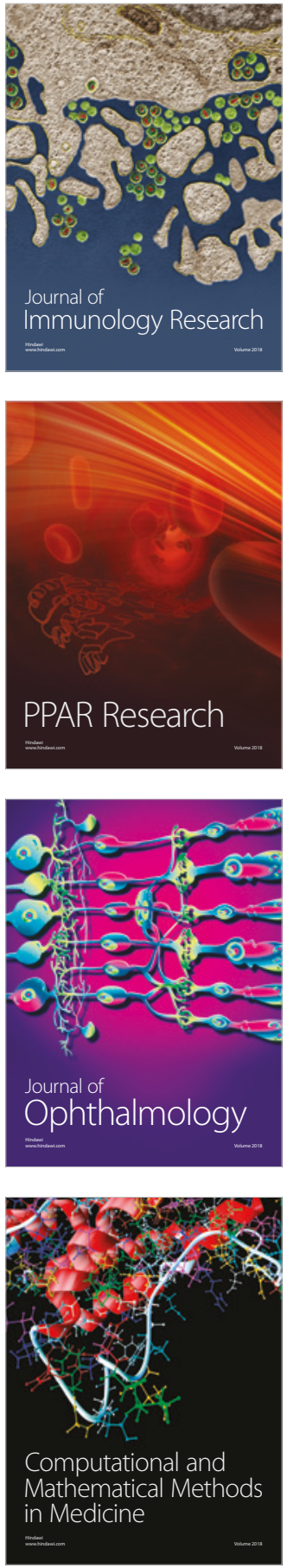

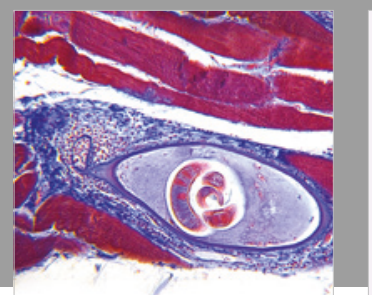

Gastroenterology Research and Practice

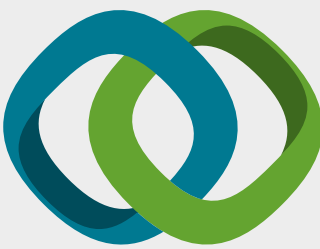

\section{Hindawi}

Submit your manuscripts at

www.hindawi.com
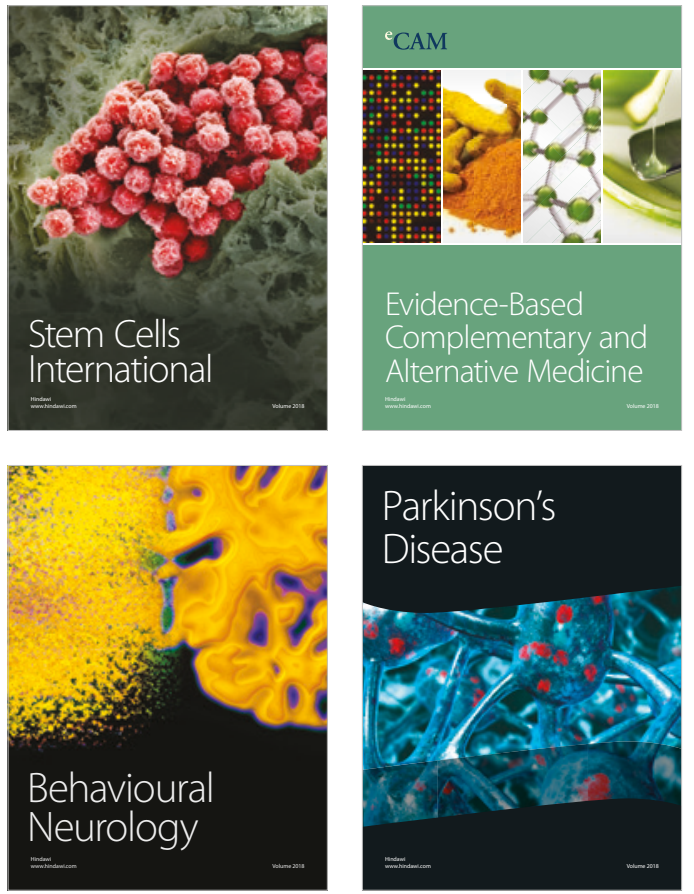

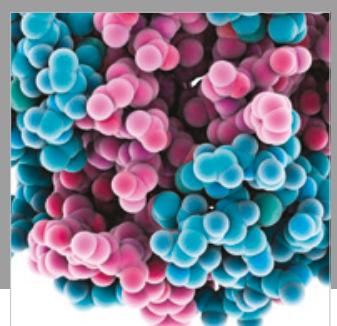

ournal of

Diabetes Research

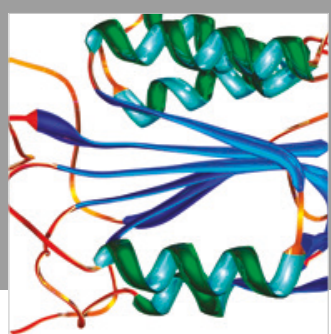

Disease Markers
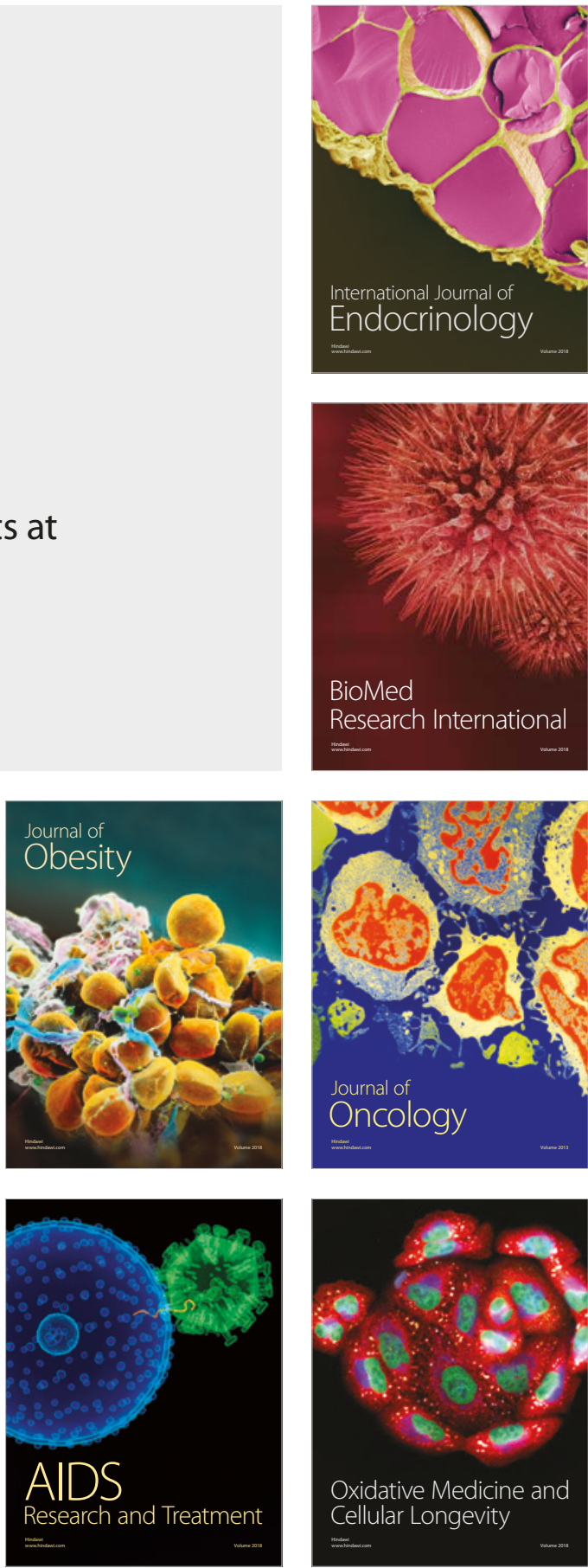